Rüdiger Bittner

\title{
Achtung und ihre moralische Bedeutung: Erwiderung auf Peter Schaber
}

\begin{abstract}
The present reply to Peter Schaber's critique of my paper Achtung und ihre moralische Bedeutung argues, first, that Schaber has no good grounds for maintaining that we have an obligation to respect every human being. Second, it explains why respect is not a fruitful attitude to take in the face of social divisions.
\end{abstract}

Peter Schabers Kritik an meinem Beitrag Achtung und ihre moralische Bedeutung will laut ihrem Titel zeigen, ,wieso moralische Achtung wichtig ist", etwas, das ich in meinem Beitrag bestritten habe. Nun ist der Ausdruck ,moralische Achtung' unglücklich, da er die Entscheidung dieses Streits vorwegnimmt: natürlich ist moralische Achtung moralisch bedeutsam. Dennoch ist klar, worüber wir reden. Nicht um die Achtung, die wir einzelnen Menschen auf Grund ihrer besonderen Eigenschaften, Fähigkeiten oder Leistungen entgegenbringen, geht es Schaber. Die moralische Bedeutung dieser Art von Achtung habe ich in meinem Artikel unterstrichen, und er erhebt dagegen keine Einwände. Ihm geht es um die Achtung, die wir nach Kants Meinung einem jeden Menschen schulden, auf die man also nicht erst durch besondere Eigenschaften, Fähigkeiten oder Leistungen einen Anspruch erwirbt. Die moralische Bedeutung dieser Art von Achtung habe ich tatsächlich bestritten, oder einfacher gesagt, ich habe bestritten, dass wir jedem Menschen Achtung schulden. Dass es doch so ist, will Schaber zeigen.

Hier ist sein Argument.

Jeder Mensch hat das Recht, selbst zu bestimmen, wie in wesentlichen Stücken sein Leben aussieht.

Jemanden achten heißt so handeln, dass der betreffende Mensch in diesem Recht nicht verletzt wird.

Also schulden wir es einem jeden Menschen, ihn zu achten.

Das Argument ist schlüssig, nur die Prämissen wecken Zweifel. Was zunächst die zweite angeht, so scheint die Wahrung eines solchen Selbstbestimmungsrechts weder notwendig noch hinreichend für das, was wir gewöhnlich ,Achtung eines Menschen' nennen. Nicht notwendig: wenn Pizarro in Beethovens Fidelio 
Florestan einkerkert, weil der ihm gefährlich werden kann, so kann man darum doch nicht sagen, er verachte oder missachte ihn. Er tut ihm Unrecht, gewiss,

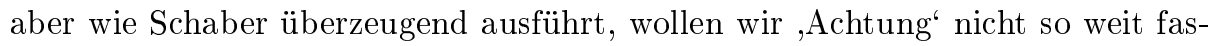
sen, dass jedes Unrecht auch schon die Achtung für denjenigen verletzt, dem es zugefügt wird. An Achtung im normalen Sinne des Wortes aber fehlt es bei Pizarro nicht. Wahrung eines Selbstbestimmungsrechts ist aber auch nicht hinreichend für Achtung: Missachtung beweist man Menschen oft mit einem Tun, von dem ihr Selbstbestimmungsrecht nicht angetastet wird. Jemanden ,fettes Schwein' oder ,blöde Kuh' nennen, ja auch schon jemanden wie Luft behandeln, ist verächtlich, aber ihr Leben selbst bestimmen können die Betreffenden darum immer noch. Man mag entgegnen, mit solchen Ausdrücken werde einem Menschen sehr wohl das Selbstbestimmungsrecht abgesprochen, was die häufig aus dem Tierreich genommenen Bezeichnungen belegen. Aber angenommen, es ist so, so reicht das nicht zu einer Bestätigung der zweiten Prämisse aus. Jemandem ein Recht absprechen, heißt noch nicht, ihn in diesem Recht, wenn er es denn hat, verletzen, und erst bei verletztem Recht auf Selbstbestimmung fehlt nach der zweiten Prämisse Achtung.

Abwegig ist die erste Prämisse. Zunächst übersieht sie, dass ein großer Teil der Dinge, die in einem Leben wesentliche Bedeutung haben, nicht Gegenstand einer Wahl sind. Wir verfügen nicht darüber, welches Geschlecht wir haben, und nur in engen Grenzen darüber, wie gut wir aussehen und mit welchen Krankheiten wir uns in welchem Alter herumschlagen müssen, und so kann von einem Recht, diese Dinge zu bestimmen, nicht die Rede sein. Wichtiger ist jedoch, dass auch dort, wo wir von einer Wahl sprechen, etwa bei Beruf, Lebenspartner und politischer Ausrichtung, so die Beispiele Schabers, wir immer darauf angewiesen sind, dass die Welt mitspielt. Wenn ich Ulrike zu meiner Lebenspartnerin erwähle, besitze ich keinerlei Recht darauf, dass sie es tatsächlich wird - vielleicht hat sie ja keine Lust. Wenn ich den Beruf des Professors der Philosophie erwähle, habe ich keinerlei Recht darauf, es auch zu werden - vielleicht gibt es davon schon genug. Gerade über das, was in einem Leben wesentliche Bedeutung hat, kann man nicht von sich aus bestimmen. In diesem Sinne sind wir nicht Herr über unser Leben. Vielmehr ist es so, ein Mensch wird einem wichtig, in eine Arbeit oder ein Fach wächst man hinein, eine politische Einstellung bildet sich aus, und wenn man Glück hat, ,landet' man mit diesen Dingen auch, in der einen oder anderen Weise. Aber all das läuft ab, wenn nicht ohne unser Zutun, so doch ohne dass wir Kontrolle darüber haben. Wenn wir aber hinsichtlich dessen, was in unserem Leben wesentlich ist, keine Verfügungsmacht besitzen, sondern diese Dinge eine Sache dessen sind, wie wir werden und was uns entgegenkommt oder eben ausbleibt, so ist es auch verfehlt, uns ein Recht darauf zuzuschreiben, dass wir wirklich werden, wozu wir uns bestimmen.

Noch ein Punkt zur Erklärung meines positiven Gegenvorschlags. Ich sagte, nicht mit der Distanz wechselseitiger Achtung sollten wir einander begegnen, sondern mit dem Mut, uns aufeinander einzulassen im Ausprobieren und Aushandeln geteilter Lebensweisen. Das Wort ,aushandeln' zu gebrauchen war ein Fehler. Denn es ließ Schaber und lässt sicher auch andere denken, ich wolle wieder einmal John Rawls' Rat der Freien, Gleichen und Vernünftigen einberufen, 
um von ihren Verhandlungen die Prinzipien unseres Zusammenlebens bestimmen zu lassen. Das ist ein Missverständnis, von diesem Gedankenspiel ist nichts Erhellendes zu erwarten. Mir geht es darum, dass die wirklichen Menschen in der Situation, in der sie stehen, und mit den Geschichten, die sie teils trennen, teils verbinden, Weisen finden, miteinander zu leben. Statt ,aushandeln' hätte ich ,sich zusammen raufen' sagen sollen. Denn damit bezeichnen wir ja den Vorgang, in dem verschiedene Menschen sich aufeinander einlassen und einander erproben und dadurch einen Weg finden, auf dem sie dann weiter und zusammen gehen können. Wenn ich das eine politische Aufgabe nannte, sollte das nicht heißen, sie gehört in die Zuständigkeit der etablierten politischen Organe. Umgekehrt, was in den etablierten politischen Organen betrieben wird, ist ein kleiner Ausschnitt jenes viel größeren Werks, an dem wir alle beteiligt sind: auszuprobieren, wie wir zusammen leben können. Dafür ist die distanzierte Haltung der Achtung unproduktiv oder hinderlich: Lebensweisen findet man nur durch zusammen leben. 